\title{
UMA ANÁLISE DAS BARREIRAS E DIFICULDADES EM LEAN HEALTHCARE
}

\section{AN ANALYSIS OF THE BARRIERS AND DIFFICULTIES IN LEAN HEALTHCARE}

\author{
Marlon Soliman* E-mail: marlon.soliman@ufrgs.br \\ Tarcisio Abreu Saurin* E-mail: saurin@ufrgs.br \\ *Universidade Federal do Rio Grande do Sul (UFRGS), Porto Alegre, RS, Brasil
}

\begin{abstract}
Resumo: O Lean Healthcare tem se tornado uma tendência de investigação devido ao potencial de resultados que pode trazer para o contexto hospitalar. No entanto, a transposição dos conceitos Lean da manufatura para a saúde ainda encontra barreiras e dificuldades que não estão totalmente esclarecidas neste novo ambiente. Assim, esta pesquisa buscou responder ao seguinte questionamento: quais as barreiras e dificuldades enfrentadas por acadêmicos e praticantes do Lean Healthcare durante tentativas de melhoria de processos hospitalares? Entrevistas semiestruturadas foram realizadas com três acadêmicos e dois praticantes do assunto, juntamente com seis rodadas de observações participantes em dois hospitais de grande porte para a coleta de dados. Os resultados obtidos foram comparados com a literatura, mostrando diferentes níveis de similaridade e contrapontos, que podem estar atrelados a fatores de contexto e aos avanços na disseminação do Lean Healthcare. Foram revelados também outros três fatores que impactam na implementação do Lean na área da saúde, e que devem ser considerados: (i) Dificuldade de experimentação; (ii) Desequilíbrio na entrega de valor; e (iii) Abordagem inadequada. Através dos exemplos mostrados, espera-se contribuir com acadêmicos e praticantes do tema, evidenciando como diferentes fatores impactam nas tentativas de melhorias de processos hospitalares e como essas barreiras podem ser contornadas.
\end{abstract}

Palavras-chave: Lean Healthcare. Barreiras. Dificuldades. Hospitais. Lean.

Abstract: Lean Healthcare has become a research trend due to the potential results that it can bring to the hospital setting. However, the tuning of Lean concepts from manufacturing to healthcare still encounter barriers and difficulties that is not entirely clear in this new environment. Thus, this research sought to answer the following question: what are the barriers and difficulties faced by researchers and practitioners of Lean Healthcare during attempts for improving hospital processes? Semistructured interviews were conducted with three researchers and two practitioners of the subject, along with six rounds of participant observation in two large hospitals to collect data. The results were compared with literature, showing different levels of similarity and counterpoints, which may be linked to context factors and advances in the spread of Lean Healthcare. It was also revealed at least three other factors that impact the implementation of Lean in healthcare, and should be considered: (i) Difficulty of experimentation; (ii) Imbalance in the delivery of value; and (iii) Inadequate approach. Through the examples shown, we expect to contribute with researchers and practitioners, showing how different factors impact on attempts to hospital process improvements and how these barriers can be circumvented.

Keywords: Lean Healthcare. Barriers. Difficulties. Hospitals. Lean.

\section{INTRODUÇÃO}

O setor de saúde compreende um elevado número de organizações prestadoras de serviços, atendendo não apenas as necessidades da população, 
mas também os requisitos de órgãos regulatórios e governamentais. Para tanto, estas organizações devem buscar a qualidade e eficiência dos serviços prestados para satisfazer as necessidades dos múltiplos stakeholders, especialmente quando considerados hospitais públicos (RODRIGUES et al., 2016).

Nesse contexto, recentemente tem-se observado um interesse crescente do setor de saúde pela utilização da produção enxuta (Lean), visando entregar maior valor aos clientes, reduzir o desperdício e proporcionar melhores condições de trabalho. O termo "Lean", introduzido no final dos anos 80 para descrever um novo sistema de produção concebido e aperfeiçoado pela Toyota Motor Company, referese a uma filosofia de gestão focada essencialmente em proporcionar valor aos clientes através de processos estáveis e regulados, com o mínimo de desperdício (WOMACK et al., 2004). A partir deste pensamento, diversas ferramentas foram desenvolvidas para operacionalizar estes conceitos, tais como o mapeamento de fluxo de valor (ferramenta para identificação de desperdícios no fluxo de materiais e informações), poka-yokes (dispositivos a prova de erros), andons (dispositivos sinalizadores de problemas), kanbans (cartões com ordens de produção para regular o mix e volume de produção), além de diversas outras (MARODIN et al., 2016).

Atualmente, os sistemas Lean evoluíram e ganharam espaço para além das fronteiras da manufatura (TEGNER et al., 2016). Especialmente a partir dos anos 2000, diversas organizações de saúde vêm buscando adaptar os conceitos do Lean à realidade de suas operações, o que na literatura ficou conhecido como Lean healthcare. As recentes publicações de Andreamatteo et al. (2015); Holden et al. (2015); Al-Balushi et al. (2014); Drotz; Poksinska (2014); De Carvalho et al. (2014); Teich; Faddoul (2013); Rinehart (2013); Burgess; Radnor (2013); Radnor et al. (2012) demonstram este fato. O Lean Healthcare é tido como uma abordagem promissora para a solução de problemas que, há muito tempo, o setor vem tentando superar, especialmente em setores de alta complexidade como cirurgia e emergência (CASTALDI et al., 2016).

A observação dos estudos publicados em Lean Healthcare, no entanto, revela alguns pontos em comum. Observa-se, inicialmente, que os casos reportados ainda concentram-se em aplicações de Lean no nível de ferramentas em departamentos ou processos específicos, e não como cultura e estratégia organizacional (HOLDEN, 2011; RADNOR et al., 2012; AL OWAD et al, 2014; DOĞAN; UNUTULMAZ, 2014; 
KAPLAN et al., 2014; GIJO; ANTONY, 2014; HAENKE; STICHLER, 2015). Como consequência, as limitações do Lean na área da saúde e as dificuldades encontradas neste novo ambiente ainda estão sendo investigadas, uma vez que o Lean Healthcare é bastante jovem quando comparado com o Lean Manufacturing (YOUNG; MACCLEAN, 2008; RANOR; WALLEY, 2008; RADNOR et al., 2012).

Diante desta situação, a pesquisa relatada neste artigo buscou responder ao seguinte questionamento: Quais as barreiras e dificuldades enfrentadas por acadêmicos e praticantes do Lean Healthcare durante tentativas de melhoria de processos hospitalares? Por se tratar de um conhecimento tácito que não pode ser obtido de forma objetiva, o assunto foi investigado através de entrevistas semiestruturadas e rodadas de observações participantes junto a equipes que estão realizando tentativas de melhorar os processos hospitalares com o Lean Thinking.

Espera-se, enquanto contribuição teórica, que este estudo avance o atual conhecimento a respeito das barreiras e limitações do Lean Healthcare, fornecendo bases que possam aumentar as chances de sucesso de implantações Lean neste ambiente.

\section{BARREIRAS E DIFICULDADES EM LEAN HEALTHCARE}

Tornar processos enxutos é um desafio em qualquer ambiente. Embora muito já se saiba sobre as dificuldades de se aplicar Lean na manufatura (EMILIANI, 2000; ACHANGA et al., 2006; SIM; ROGERS, 2009; BHASIN, 2012; COPETTI et al., 2016) as barreiras, limitações e adaptações necessárias no setor hospitalar ainda guardam lacunas de investigação.

Grove et al. (2010) identificaram seis desafios para aplicar Lean em um serviço público de cuidado primário na Inglaterra: (a) alta variabilidade do processo; (b) falta de entendimento do Lean; (c) comunicação e liderança deficientes; (d) foco em metas nacionais; (e) problemas na definição de perdas; e (f) problemas na identificação dos clientes e seus requisitos de valor. Os autores afirmam que a chave para contornar estas dificuldades é estabelecer uma visão compartilhada de longo prazo, pois não há solução rápida e fácil para a melhoria de produtividade.

Brandão de Souza; Pidd (2011), por sua vez, reportam oito barreiras encontradas durante aplicações de Lean em hospitais públicos: (a) percepção; (b) 
terminologia; (c) habilidades pessoais/profissionais; (d) momento organizacional; (e) silos funcionais e profissionais; (f) hierarquia e gerência; (g) coleta de dados e medição de desempenho; (h) resistência à mudança/ceticismo. Os autores argumentam que as barreiras (a), (c), e (f) exercem papeis importantes apenas nas aplicações de Lean na saúde, não estando presentes na manufatura. Já as barreiras (b), (d), (e), (g) e (h) são comuns tanto na saúde como na manufatura, ainda que se apresentem em intensidades diferentes para cada cenário.

Os estudos de Radnor et al. (2012) junto a hospitais públicos ingleses identificaram quatro fatores chaves que dificultam a aplicação do Lean neste ambiente: (a) definição do cliente; (b) aplicação desarticulada; (c) aplicação baseada em ferramentas; e (d) dificuldade de sustentação a longo prazo. Para os autores, estas dificuldades são decorrentes das aplicações Lean restritas ao nível de ferramentas, que produz resultados rápidos, mas limitados a uma pequena porção do sistema.

Al-Balushi et al. (2014), após realizarem uma varredura na literatura disponível, identificaram sete "fatores de prontidão" que devem ser desenvolvidos pelos hospitais antes de se lançarem em uma jornada Lean: (a) apoio da equipe de liderança; (b) alinhamento estratégico; (c) identificação dos valores e grupos de clientes; (d) identificação dos processos para eliminação de desperdícios; (e) treinamento e envolvimento das pessoas nos princípios e métodos Lean; (f) sistema de medição e recompensas alinhado aos objetivos Lean; e (g) nivelamento da demanda e da capacidade para melhorar o fluxo. Os autores concluem que o não atendimento a esses fatores pode frustrar as chances de sucesso das aplicações Lean, mas reforçam a necessidade de dados empíricos para confirmar esta hipótese.

Mais recentemente, Andreamatteo et al. (2015) realizaram uma revisão sistemática sobre o atual desenvolvimento do Lean Healthcare. Os autores classificaram os estudos em teóricos ou empíricos, e dentro dos grupos dos teóricos, analisaram as principais referências que tratam sobre as barreiras e dificuldades em Lean Healthcare. 


\section{METODOLOGIA}

Esta pesquisa foi realizada conforme o fluxo metodológico mostrado na Figura 1, compreendendo quatro macros etapas: Referencial teórico; Coleta de dados; Descrição e análise dos resultados; e Discussão dos resultados.

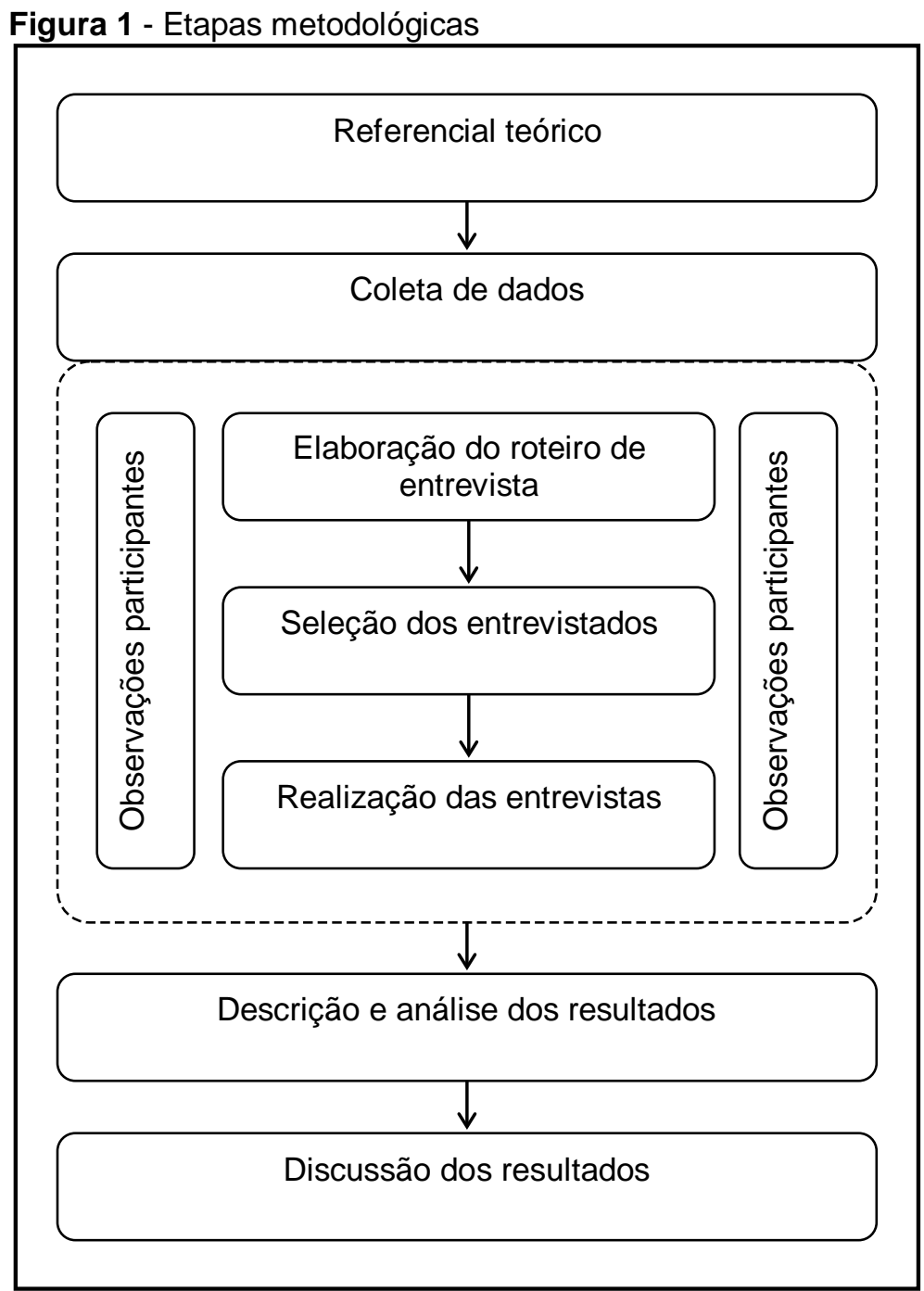

O referencial teórico teve como objetivo estabelecer o estado da arte sobre a temática de barreiras e dificuldades em Lean Healhcare. Para isso, utilizou-se do levantamento realizado por Andrematteo et al. (2015), além da busca em portais científicos (Emerald Insight; Scopus e Science Direct) com combinações das palavras chaves "Lean"; "Lean Healthcare"; "Lean health care"; "barriers"; e "difficulties". Dez artigos foram selecionados pela sua relevância: Brandão de Souza (2009); Grove et al. (2010); Poksinska (2010); Brandão de Souza; Pidd (2011); 
Radnor (2011); Burges; Radnor (2012); Morrow et al. (2012); Radnor et al. (2012); Davis; Adams (2012) e Al-Balushi et al. (2014).

A etapa seguinte envolveu a coleta de dados. Devido à característica exploratória da pesquisa, optou-se pela utilização de uma abordagem qualitativa, através de entrevistas semiestruturadas e observações participantes. Um roteiro contendo oito questões abertas foi elaborado para garantir a cobertura dos principais eixos da pesquisa, mas mantendo-se a flexibilidade para explorar outros temas de interesse ao longo das entrevistas.

A seleção dos entrevistados foi realizada de forma intencional, pelo histórico de trabalhos prévios e acessibilidade das pessoas. A amostra foi composta por cinco entrevistados, sendo três acadêmicos e dois praticantes. As entrevistas foram realizadas de forma presencial e por videoconferência, e os dados registrados em áudio e em notas. Somadas, as entrevistas duraram aproximadamente 225 minutos.

Para as observações participantes, os pesquisadores inseriram-se em times que estão realizando aplicações Lean na reformulação e melhoria de processos hospitalares. O papel dos pesquisadores foi atuar como um colaborador externo, fornecendo orientações para a implantação das melhorias e aconselhamento técnico. Foram realizadas seis rodadas de observações, contemplando dois processos em dois hospitais de grande porte, totalizando aproximadamente 13 horas de observações. Os dados foram registrados em notas de campo.

$\mathrm{Na}$ etapa de descrição e análise dos resultados, foi realizada a caracterização da amostra selecionada, tanto dos entrevistados quanto das observações participantes. Posteriormente, os dados foram analisados de acordo com a estrutura mostrada na Figura 2. 
Figura 2 - Estrutura para análise de dados

FONTES DE EVIDÊNCIA

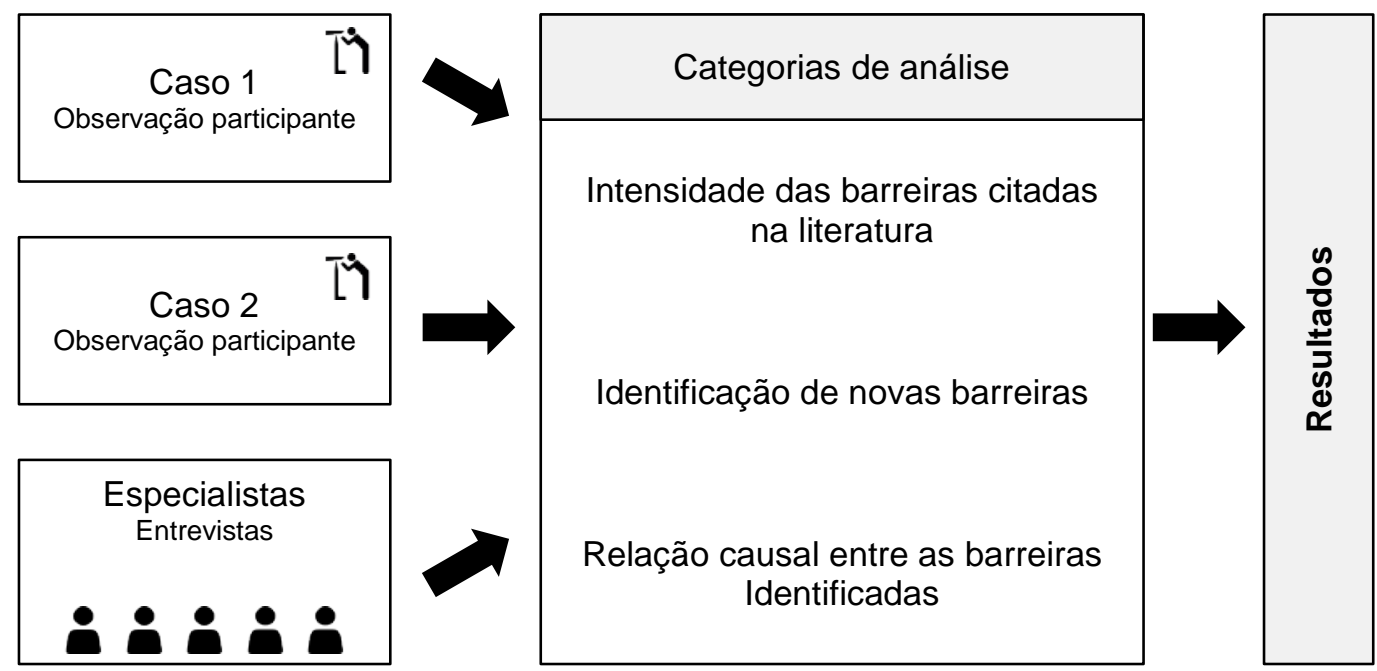

Conforme o fluxo mostrado na Figura 2, as fontes de evidências utilizadas foram analisadas de acordo com três categorias. Inicialmente, uma comparação com as barreiras identificadas no referencial teórico foi realizada, onde cada fonte de evidência foi contrastada com a lista de barreiras, verificando-se a intensidade das mesmas. Deve-se ressaltar que tanto as observações participantes quanto às entrevistas constituem-se de fontes de dados onde a percepção de barreiras advém de contextos e situações diferentes. O propósito desta diferenciação é justamente contribuir na identificação de barreiras que sejam associadas às características comuns de ambientes hospitalares, buscando-se similaridades entre diferentes tentativas de intervenção com base no Lean.

Posteriormente, utilizou-se tanto das notas das observações quanto de fragmentos das entrevistas para a identificação de outras barreiras além daquelas referenciadas na literatura, através da análise do discurso. Por último, uma matriz contendo todas as barreiras identificadas foi elaborada, e as relações entre as mesmas foram identificadas com base nos dados coletados tanto através da observação participante quanto das entrevistas com especialistas. Estas relações foram utilizadas na construção de um mapa conceitual, que mostra graficamente como essas barreiras se reforçam/atenuam. 


\section{DESCRIÇÃO E ANÁLISE DOS RESULTADOS}

O Quadro 1 apresenta o perfil dos entrevistados selecionados para comporem a amostra da pesquisa. Para preservar a identidade dos respondentes e possíveis conflitos de interesse, utilizou-se do código " $\mathrm{A}_{i}$ " para aqueles cujo envolvimento com Lean Healthcare é basicamente através de estudos acadêmicos, e "Pi" para os profissionais que atuam em consultorias ou como colaboradores de hospitais.

Quadro 1 - Perfil dos entrevistados

\begin{tabular}{|c|l|}
\hline Código & \multicolumn{1}{c|}{ Perfil } \\
\hline A1 & $\begin{array}{l}\text { Prof. Dr. em Engenharia de Produção, com 10 anos de experiência em Lean no setor } \\
\text { automotivo. Atualmente, atua em um projeto de aplicação de Lean no Centro de Materiais } \\
\text { e Esterilização (CME) de um hospital de grande porte. }\end{array}$ \\
\hline A2 & $\begin{array}{l}\text { Profa. MSc em Engenharia de Produção e Sistemas. Realizou pesquisas de revisão } \\
\text { sistemática para explorar o estado da arte do Lean Healthcare, cobrindo aproximadamente } \\
300 \text { artigos publicados. }\end{array}$ \\
\hline A3 & $\begin{array}{l}\text { Mestranda em Engenharia de Produção, com foco em Lean Healthcare. Atualmente atua } \\
\text { em projetos junto aos setores de Emergência, Farmácia, Centro de Esterilização e } \\
\text { Materiais (CME) e Radiologia de um hospital de grande porte. }\end{array}$ \\
\hline P1 & $\begin{array}{l}\text { Diretor de uma consultoria especializada em Lean. Possui mais de 20 anos de vivência em } \\
\text { Lean, e aproximadamente 7 anos em Lean Healthcare. Possui experiências em hospitais } \\
\text { de Minas Gerais, Distrito Federal, Rio Grande do Sul, São Paulo, Rio de Janeiro e Manaus. }\end{array}$ \\
\hline P2 & $\begin{array}{l}\text { Engenheiro de Produção. Atua desde 2013 como especialista de Gestão de Inovação e } \\
\text { Processos em um hospital de grande porte, liderando processos de melhoria com base no } \\
\text { Lean. }\end{array}$ \\
\hline
\end{tabular}

Além das entrevistas, a coleta de dados contemplou incursões em dois times que estão fazendo tentativas de melhorias de processos hospitalares segundo abordagem Lean. O Quadro 2 apresenta uma breve descrição dos cenários onde foram realizadas as observações, codificadas como "O1" e "O2".

Quadro 2 - Descrição das observações participantes

\begin{tabular}{|c|l|}
\hline Código & \multicolumn{1}{|c|}{ Descrição } \\
\hline O1 & $\begin{array}{l}\text { Acompanhamento de reuniões da equipe focada na melhoria do processo de transferência } \\
\text { do paciente clínico da emergência para as unidades de internação em um hospital público } \\
\text { de grande porte utilizando Lean. }\end{array}$ \\
\hline O2 & $\begin{array}{l}\text { Acompanhamento da equipe focada na melhoria do processo de alta da maternidade em } \\
\text { um hospital privado de grande porte utilizando Lean. }\end{array}$ \\
\hline
\end{tabular}


A análise das respostas dos entrevistados e as percepções extraídas dos grupos $\mathrm{O} 1$ e $\mathrm{O} 2$ foram classificadas e comparadas aos estudos selecionados durante o referencial teórico, conforme mostrado no Quadro 3. Utilizou-se como escala a simbologia "+" para barreiras ou dificuldades que fortemente impactam a aplicação do Lean Healthcare; "+/-“ para barreiras intermediárias ou que dependem de determinadas condições; "“" para barreiras leves ou facilmente contornáveis; e "X" para os fatores citados na literatura como barreiras, mas que não se manifestaram nas experiências dos respondentes ou nas observações O1 e O2. Os campos em branco referem-se a situações que, com base nos relatos e observações, não foi possível concluir sobre a incidência dos fatores.

Quadro 3 - Análise das barreiras e dificuldades relatadas pelos entrevistados e observadas em O1 e O2

\begin{tabular}{|c|c|c|c|c|c|c|c|c|c|}
\hline \multicolumn{2}{|r|}{ Barreiras e dificuldades } & Referência & A1 & A2 & A3 & P1 & P2 & 01 & 02 \\
\hline F1 & Alta variabilidade do processo & [1], [5] & $+1-$ & + & + & $+1-$ & $+1-$ & + & - \\
\hline $\mathrm{F} 2$ & Falta de entendimento do Lean & $\begin{array}{c}\text { [1], [4], [5], } \\
{[9]}\end{array}$ & & + & - & + & - & - & \\
\hline F3 & Foco em metas nacionais (centrais) & [1]; [7] & $\mathrm{X}$ & $x$ & $\mathbf{x}$ & $x$ & $\mathbf{x}$ & $\mathrm{x}$ & $\mathrm{x}$ \\
\hline $\mathrm{F} 4$ & Dificuldade na definição de perdas & {$[1],[5]$} & - & $+1-$ & + & $+1-$ & - & $+1-$ & + \\
\hline F5 & $\begin{array}{l}\text { Dificuldade de entender quem é o } \\
\text { cliente e o que ele valoriza }\end{array}$ & $\begin{array}{l}\text { [1], [3], [4], } \\
\text { [8], [9], [10] }\end{array}$ & - & + & $+1-$ & & $x$ & - & $+1-$ \\
\hline F6 & Percepção errônea do Lean & [2], [9] & $\mathrm{X}$ & & $+1-$ & $+1-$ & - & $\mathrm{x}$ & $\mathrm{x}$ \\
\hline $\mathrm{F} 7$ & Terminologia & [2] & $\mathrm{x}$ & & - & $+1-$ & $x$ & $x$ & $\mathrm{X}$ \\
\hline F8 & $\begin{array}{l}\text { Habilidades pessoais/profissionais } \\
\text { incompatíveis com o Lean }\end{array}$ & [2], [9] & & + & + & + & $+l-$ & $+I-$ & \\
\hline F9 & Sustentação em longo prazo & $\begin{array}{c}\text { [2], [3], [6], } \\
{[8]}\end{array}$ & & & + & + & + & & \\
\hline F10 & Silos funcionais e profissionais & [2], [9] & $+1-$ & + & + & + & & $+1-$ & $+1-$ \\
\hline F11 & $\begin{array}{l}\text { Hierarquia e gerência } \\
\text { incompatíveis com o Lean }\end{array}$ & $\begin{array}{c}1],[2],[4], \\
{[9],[10]}\end{array}$ & + & + & + & + & + & & \\
\hline $\mathrm{F} 12$ & $\begin{array}{l}\text { Dificuldade de coleta de dados e } \\
\text { medição de desempenho }\end{array}$ & [2], [8] & + & & $+l-$ & $+l-$ & + & + & + \\
\hline F13 & Resistência a mudança/ceticismo & [2], [8] & & - & + & $+1-$ & $+1-$ & $+1-$ & \\
\hline F14 & Aplicação desarticulada & [3], [4], [10] & & + & $+1-$ & $+1-$ & & & \\
\hline F15 & Aplicação baseada em ferramentas & [3] & - & & & $+1-$ & & - & + \\
\hline F16 & Complexidade do processo de implantação & [8] & + & & + & $+1-$ & + & + & - \\
\hline
\end{tabular}

Legenda: (+) Barreira forte (+/-) Barreira intermediária (-) Barreira fraca $(X)$ Barreira ausente.

Fonte: Elaborado com base em [1] Grove et al. (2010); [2] Brandão de Souza; Pidd (2011); [3] Radnor et al. (2012); [4] Al-Balushi et al. (2014); [5] Brandão de Souza (2009); [6] Davis; Adams (2012); [7] Burges; Radnor (2012); [8] Morrow et al. (2012); [9] Poksinska (2010); [10] Radnor (2011)

A partir do Quadro 3, observa-se que o fator "F11 Hierarquia e gerência incompatíveis com o Lean" foi o que mais vezes apresentou classificação como 
barreira forte. Mesmo não sendo possível constatar a influência deste fator nas observações participantes realizadas, todos os cinco entrevistados citaram este ponto como um entrave relevante para o Lean Healthcare.

Na opinião de A1, a constatação de que os papéis hierárquicos e gerenciais dentro dos hospitais tornam-se uma barreira é decorrente dos modelos de gestão adotados, especialmente na comparação entre hospitais públicos e privados. Na sua experiência, os hospitais públicos carecem de uma gestão mais próxima do nível empresarial, com definições de responsabilidades e atribuições, estratégia e metas mais claras. Com isso, os esforços de melhorias de processos encontram dificuldades para serem implementados, pois não há bases gerenciais para suportálos.

Sobre esse tema, soma-se a opinião de A3. A entrevistada vê como empecilhos para o Lean Healthcare a tradição de hospitais de promoverem os melhores médicos para cargos gerenciais, sem que necessariamente este profissional esteja capacitado para isso. A situação agrava-se quando estes profissionais não estão dedicados à nova função, e cita como exemplo: "Hoje, o gestor principal do Hospital [nome removido] é responsável por um orçamento de quase $\mathrm{R} \$ 1$ bi por ano. Mas ele também é um cirurgião, e tem seus problemas como tal".

Depois de "F11", aparecem empatados na segunda posição os fatores "F12 Dificuldade de coleta de dados e medição de desempenho" e "F16 Complexidade do processo de implantação" como os que mais receberam pontuações de forte barreira. Sobre a coleta de dados, P2 traz à tona uma questão relevante, ao citar que os hospitais possuem registros de dados prioritariamente para a rastreabilidade do cuidado, sendo quase ausentes os dados de produção. Não é comum nesse ambiente a coleta de dados para se verificar o desempenho dos processos, e isso aparece como uma barreira.

A opinião de P1 corrobora essa afirmação. Segundo o entrevistado "dados, fatos e números não fazem parte do repertório de gestão do dia-a-dia dos hospitais". Essa foi também a realidade encontrada por A1. Ao ser questionado sobre os resultados atingidos no projeto em que atua, o entrevistado comentou que as melhorias são perceptíveis, mas não possui números para isso, pois houve dificuldade de incorporar as medições de desempenho nas rotinas da equipe. 
A complexidade da implantação foi frequentemente mencionada pelos entrevistados, e este ponto ficou claro também nas rodadas de observação O1. Como citado por A3, "na saúde aparece mais a imprevisibilidade e a variabilidade de tratamentos e exames necessários. Isso faz com que seja mais complexo de ser mapeado e compreendido, exigindo muito mais horas de tentativa de se entender o sistema do que na manufatura". Durante as observações de 01 , foi possível perceber a relevância dessa informação, pois o mapeamento do fluxo de valor para o processo em questão assumiu dimensões espantosas, com muitas atividades envolvidas e a participação de diversos agentes.

Ainda assim, P1 contrapõem que a complexidade de implementação seja uma barreira rígida para o Lean Healthcare. Segundo ele "o nível de complexidade de uma fábrica, em termos de número de variáveis, é tremendamente maior do que um hospital. A diferença é que o hospital depende muito mais das pessoas, e a fábrica depende mais de máquinas e equipamentos. São coisas diferentes".

$\mathrm{Na}$ terceira posição, "F1 Alta variabilidade dos processos"; "F8 Habilidades pessoais/profissionais incompatíveis com o Lean"; "F9 Sustentação em longo prazo"; e "F10 Silos funcionais e profissionais" aparecem empatados na análise dos fatores que mais vezes receberam classificação de barreira forte. Em relação a $F 1$, no entanto, constata-se que a questão de variabilidade nos processos é relativa, pois existem processos com diferentes níveis de variabilidade dentro de hospitais, e a opinião dos entrevistados pode estar condicionada a experiências prévias.

Os exemplos de intervenção citados por A1 e P2 são provas disso. O primeiro, que baseou sua resposta no caso da aplicação de Lean em um Centro de Materiais e Esterilização, relatou que a demanda por esses materiais segue um padrão conhecido em função do agendamento do bloco cirúrgico (principal cliente), e por isso a variabilidade é controlável. P2 citou a experiência de uso de Lean no setor de preparo das refeições, que embora sofram variabilidade, isso não chega a ser um entrave maior na implementação do Lean.

As habilidades dos profissionais da saúde foi algo enfatizado pelos respondentes. Para $A 3$, os problemas começam na formação desses profissionais, pois eles são pouco expostos à gestão. Por isso, quando se entra com a abordagem enxuta, essa deficiência costuma ser um agravante. A3 afirma: "O Lean é uma visão diferenciada de gestão. Os médicos e enfermeiros normalmente não possuem visão 
de gestão. Então como falar de uma gestão diferenciada sem eles nem compreenderem a gestão básica?".

A sustentação em longo prazo (F9) trata de uma dificuldade que não é exclusiva da saúde, mas manifesta-se em todos os setores. Conforme as experiências de P1, é natural que isso aconteça, porque "Lean não é fácil, e as chances de não dar certo são maiores". Nesse ponto, A3, P1 e P2 concordam que as chances de haver sustentação em longo prazo são melhores quando a alta administração efetivamente "compra" a ideia do Lean e trata-o com a atenção que necessita. Se não for assim, o decaimento é esperado.

A questão sobre silos profissionais (F10) trouxe à tona uma barreira complexa do sistema. A entrevistada A3, ao reportar a situação de um hospital, afirmou "não haver silos, e sim feudos profissionais", referindo-se ao alto isolamento entre as diversas subestruturas hospitalares. Isso força o aparecimento de melhorias locais, mas que não necessariamente melhoram o sistema como um todo. A2 ressalta que "o hospital ainda é um sistema muito complexo. As pessoas não sabem bem como fazer ainda para cortar através dos silos", demonstrando que esse ponto ainda precisa ser sanado com mais estudos.

Ao se analisar os fatores que mais vezes foram enquadrados como barreiras intermediárias, observa-se um empate entre "F1 Alta variabilidade dos processos"; "F4 Dificuldade na identificação de perdas"; "F10 Silos profissionais"; e "F13 Resistência à mudança". Em relação a F1, no entanto, consta-se que a questão de variabilidade nos processos é relativa, pois existem processos com diferentes níveis de variabilidade dentro de hospitais, e a opinião dos entrevistados pode estar condicionada a experiências prévias.

Os exemplos de intervenção citados por A1 e P2 são provas disso. A1, que relatou o caso da aplicação de Lean em um Centro de Materiais e Esterilização, cita que a demanda por esses materiais segue um padrão conhecido em função do agendamento do bloco cirúrgico (principal cliente), e por isso a variabilidade é controlável. P2 citou a experiência de uso de Lean no setor de preparo das refeições, que embora sofram variabilidade, isso não chega a ser um entrave maior na implementação do Lean.

A dificuldade de identificação das perdas também depende do processo analisado. Durante as observações de 02 , notou-se que a destreza da equipe 
assistencial em realizar as tarefas rotineiras encobria parte das perdas do sistema. As pessoas haviam aprendido a realizar tarefas desnecessárias de uma maneira tão eficiente que foi difícil enxergar onde as perdas ocorriam.

No extremo inferior, a falta de entendimento do Lean (F2) foi o fator que menos aparentou gerar preocupação ou impedir as tentativas de melhorias de processos usando Lean. Isso porque, como destaca A3 "Lean é uma jornada de aprendizado", ou seja, não se espera que as organizações partam de um estágio inicial com pleno domínio do assunto, mas sim que evoluam com a melhoria contínua. P1 reforça: "É necessário que haja um aprendizado em um primeiro ciclo, para que as pessoas entendam e sejam capazes de replicar isso, porque é diferente do que elas fazem no dia-a-dia".

Isso fica ainda mais claro na experiência de P2. O entrevistado relatou uma situação onde se fez uma extensa capacitação da equipe em um primeiro momento, incluindo treinamentos formais e leituras de obras referências no assunto. Mesmo assim, o Lean não se concretizou, pois as pessoas tiverem dificuldades na aplicação prática dos conceitos, o que sugere que existem fatores mais fortes do que a falta de entendimento.

Por último, o fator "F3 Foco em metas nacionais (centrais)" revela uma situação exclusiva para as aplicações dentro do sistema inglês de saúde pública, o National Health System (NHS). Barreiras impostas por esse fator não foram constatadas entre os entrevistados e nas observações realizadas.

\section{DISCUSSÃO DOS RESULTADOS}

Em um primeiro momento, a análise dos dados buscou identificar o grau de semelhança entre as barreiras do Lean Healthcare reportadas na literatura e aquelas vivenciadas por acadêmicos e praticantes em hospitais brasileiros. Ao decorrer do estudo, no entanto, foram identificados outros três fatores que impactam a utilização da mentalidade enxuta no setor hospitalar, e que não estão citados nos artigos consultados. Esses fatores são descritos nas seções 5.1, 5,2 e 5.3. Por último, a seção 5.4 analisa a inter-relação entre os fatores identificados na literatura e os levantados pelo estudo. 


\subsection{Dificuldade de experimentação}

Processos que envolvem pacientes são muito mais delicados do que a simples transformação de uma matéria-prima. Por isso, a modificação de processos na área da saúde precisa ser feita com maior atenção e previsibilidade. Essa é uma dificuldade adicional que se encontra no Lean Healthcare quando compara-se com a manufatura, mas que não é vista como negativa. Sobre isso, A3 afirma: "Como eu tenho essa visão de Engenharia de Resiliência e complexidade, eu não me sinto segura sem fazer uma abordagem muito profunda do estado atual. O Lean não quer muito saber como está o estado atual, quer partir logo para a ação. Mas na saúde, é mais complicado, se a gente mapear um processo mal e fizer uma implementação sem pensar muito no contexto da variabilidade envolvida, pode-se matar pessoas".

Como muitos dos estudos de Lean Healthcare estão sendo iniciados pelas áreas industriais dos hospitais (alimentação, farmácia, faturamento, entre outros), essa é uma barreira que possivelmente ainda não tenha sido experimentada por muitas equipes. É necessário que os acadêmicos e profissionais, ao aprofundaremse na jornada Lean, estejam conscientes de que a análise do estado atual de processos hospitalares necessita um grande esforço e tempo. É também necessário incluir opinião de diversas pessoas, buscando-se o melhor caminho de ação, sempre tendo como prioridade absoluta a segurança do paciente.

\subsection{Desequilíbrio na entrega do valor}

A literatura postula como uma barreira para o Lean Healthcare a dificuldade de identificação dos clientes e seus requisitos de valor. No entanto, a preocupação mostrada pelos entrevistados revela algo diferente. A questão central não é quem são os clientes, mas sim como equilibrar o valor entre todos os stakeholders que atuam nos processos. Segundo A2, "é necessário haver uma ponderação para atender os requisitos de todos os clientes, sejam pacientes, médicos, equipes de enfermagem e planos de saúde". Para A3, o sistema de saúde "foi desenvolvido em torno das necessidades dos médicos". Para P2 "o modelo dos hospitais hoje é médico-centrado. É necessário que se comece a trabalhar em um modelo centrado no paciente, para que esse tenha uma experiência diferenciada". 
Assim, constata-se que este desequilíbrio sobre a entrega do valor, que está enraizado no sistema, atua como uma barreira para o Lean Healthcare. Quando os processos forem reformulados visando uma melhor distribuição do valor e trazendo a figura do paciente para um patamar de mais alto nível, espera-se que se encontre resistência por parte das equipes, naturalmente acostumadas a defender seus interesses sob a forma de silos profissionais.

\subsection{Abordagem inadequada}

Os relatos dos entrevistados apontam que a abordagem ou a forma como o Lean é introduzido nas organizações de saúde é um ponto crítico que irá condicionar o sucesso da implementação. As experiências vividas por A3 e P1 mostram que, ao se utilizar exemplos da manufatura ou conceitos desconectados da realidade dos hospitais como forma de ensinar Lean, aumenta-se o risco de se criar resistências por parte da equipe de assistência.

A3 exemplifica este fato ao citar a participação em um treinamento para uma equipe de enfermagem onde o ministrante utilizou dados de fresadoras para explicar controle estatístico de processos. Segundo $A 3$, isso gerou uma reação muito adversa da equipe, que não se sentiu motivada a colaborar. P1 afirma que "o grande desafio é conduzir uma intervenção fazendo com que isso seja bacana, interessante e inteligente para as pessoas". A3 ainda completa: "A forma como eu vejo para se inserir (o Lean) mais fácil, para ficar mais palatável para eles é falar sobre a segurança do paciente. Não trago em nenhum momento exemplos de manufatura, e tento evitar ao máximo de falar de carros".

Isso justifica não se ter visualizado "F6 Percepção errônea do Lean" e "F7 Terminologia" como barreiras fortes ao longo do estudo. Atualmente, cases e artigos de Lean Healthcare estão muito mais acessíveis. Portanto, não há motivos para insistir no uso de uma abordagem de manufatura no ambiente hospitalar.

\subsection{Análise da inter-relação entre os fatores}

A análise do relacionamento entre as barreiras presentes em iniciativas de Lean Healthcare foi realizada através da construção de um mapa conceitual (Figura 
3), onde dois rótulos foram utilizados: "causa", quando existe relação direta de causa-efeito entre as barreiras na direção da seta; e "amplifica" quando a barreira antecessora não causa a sucessora, mas seus efeitos amplificam o efeito da conseguinte.

Figura 3 - Mapa conceitual da inter-relação entre as barreiras

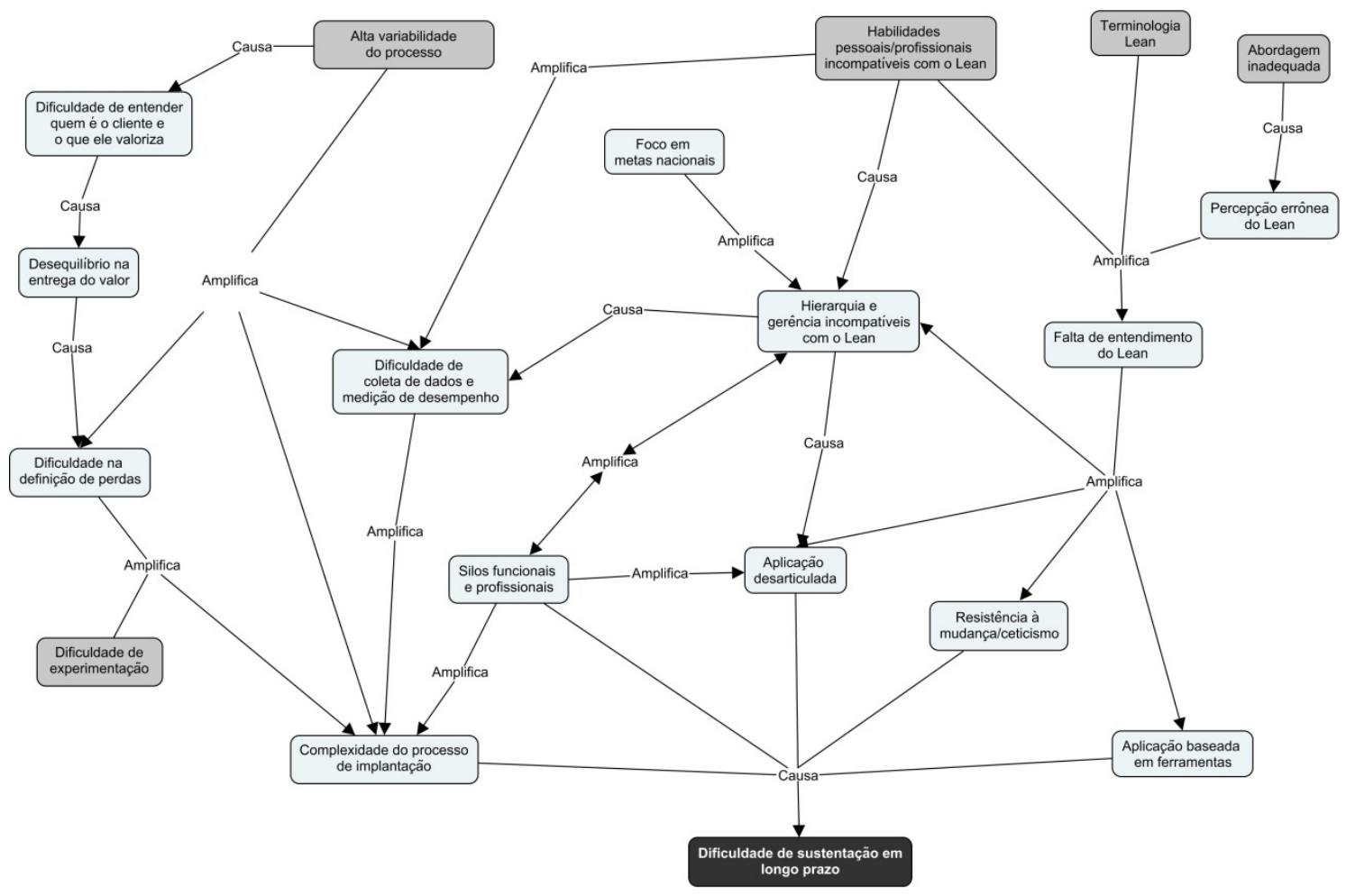

A partir da Figura 3, nota-se primeiramente que o fator "F9 Sustentação em longo prazo" é o objetivo final da implantação Lean. Por isso, essa barreira é tratada como consequência de todas as demais, e não como uma barreira em si.

Em segundo lugar, as barreiras "Dificuldade de experimentação" e "Abordagem inadequada" exercem um papel periférico na análise das barreiras. No entanto, enquanto o efeito da primeira é de amplificar a complexidade do processo de implantação, percebe-se que a utilização de uma abordagem inadequada é um dos causadores da percepção errônea do Lean, conforme já destacado na seção 5.3 .

O fator "F11 Hierarquia e gerência incompatíveis com o Lean", que mais vezes apresentou classificações como barreira forte, destaca-se no mapa devido ao seu papel central na conexão entre as barreiras, tendo como principal causa o fator 
"F8 Habilidades pessoais/profissionais incompatíveis com o Lean". A baixa qualificação profissional em gestão na saúde (sob a ótica Lean) leva a gerências a adotarem ações incompatíveis com a filosofia, sendo uma das principais causas de fracasso das implantações enxutas. Adicionalmente, a presença de silos funcionais, foco em metas nacionais e falta de entendimento do Lean amplificam os efeitos desse fator.

Por último, recebe destaque também a complexidade do processo de implantação. Embora não se possa atribuir causas específicas, a complexidade no setor hospitalar é decorrente do elevado número de interações dinâmicas entre os componentes do sistema. Diferente dos sistemas produtivos industriais (ex: linhas de montagem), hospitais são sistemas abertos onde as singularidades de cada paciente precisam ser tratadas, racionalizando-se recursos materiais e humanos. Nesse contexto, a variabilidade presente, a indisponibilidade de dados, a dificuldade de experimentação e a presença de silos funcionais são fatores que contribuem para o aumento da complexidade, e que devem ser atenuados para que a implantação Lean tenha mais chances de sucesso.

\section{CONCLUSÃO}

A análise dos resultados obtidos revela que as dificuldades e barreiras enfrentadas durante a aplicação do Lean Healthcare possuem diferentes graus de importância, que variam de acordo com os contextos. Com exceção dos fatores "F3 foco em metas nacionais (centrais)"; "F9 Sustentação em longo prazo" e "F11 Hierarquia e gerência incompatíveis com o Lean", todos os demais fatores apresentaram diferentes níveis de importância entre os respondentes. Isso pode ser atribuído ao background de cada um, pois parte da amostra possui experiências em processos envolvendo pacientes, e parte atuou em áreas industriais dentro do contexto hospitalar.

O estudo também mostra que as barreiras referenciadas na literatura não são estáticas. Fatores como "F6 Percepção errônea do Lean" e "F7 Terminologia" podem ter deixado de serem barreiras fortes com a popularização do Lean Healthcare, tornando o assunto mais próximo da realidade médico-hospitalar. Por outro lado, é 
possível que surjam novas barreiras conforme o Lean Healthcare evolua. É necessário que se trate o tema de maneira dinâmica.

De modo geral, os resultados encontrados apresentaram diferentes níveis de similaridade com o atual estado da arte. Revelaram-se contrapontos em relação ao que se entende atualmente como barreiras e dificuldades em Lean Healthcare, e apontaram-se no mínimo outros três fatores que impactam na implementação do Lean na área da saúde.

O estudo é limitado pelo número de participantes envolvidos e pelo fato dos processos observados estarem em estágios iniciais de desenvolvido. Por isso, não se pode concluir ainda sobre o ranking ou a ponderação de importância de cada um dos fatores. Também não se pode afirmar com precisão quais itens deixaram de ser barreiras e quais deveriam sem incluídos na lista. No entanto, os resultados obtidos são indicativos de que há necessidade de maior investigação no tema, abrindo-se caminho para pesquisas que incluam fatores de contexto e análises quantitativas.

\section{REFERÊNCIAS}

ACHANGA, P.; SHEHAB, E.; ROY, R.; NELDER, G. Critical success factors for lean implementation within SMEs. Journal of Manufacturing Technology Management, v. 17, n. 4, p. 460-471, 2006. http://dx.doi.org/10.1108/17410380610662889

AL-BALUSHI, S.; SOHAL, A.S.; SINGH, P.J.; AL HAJRI, A.; AL FARSI, Y.M.; AL ABRI, R. Readiness factors for lean implementation in healthcare settings -a literature review. Journal of Health Organization and Management, v. 28, n. 2, p. 135-153, 2014. http://dx.doi.org/10.1108/JHOM-04-2013-0083

AL OWAD, A.; KARIM, M.A.; MA, L. Integrated Lean Six Sigma Approach for Patient Flow Improvement in Hospital. Emergency Department. Research in Materials and Manufacturing Technologies, v. 835, p. 1893-1902, 2014. http://doi.org/10.4028/www.scientific.net/AMR.834-836.1893

ANDREAMATTEO, A.; IANNI, L.; LEGA, F.; SARGIACOMO, M. Lean in healthcare: a comprehensive review. Health Policy, in press. 2015.

http://doi.org/10.1016/j.healthpol.2015.02.002

BHASIN, S. Prominent obstacles to lean. International Journal of Productivity and Performance Management, v. 61, n. 4, p. 403-425, 2012. http://www.emeraldinsight.com/doi/abs/10.1108/17410401211212661

BRANDÃO DE SOUZA, L. Trends and approaches in Lean Healthcare. Leadership in Health Services, v. 22, n. 2 2009, p. 121-139, 2009.

http://www.emeraldinsight.com/doi/abs/10.1108/17511870910953788 
BRANDÃO DE SOUZA, L.; PIDD, M. Exploring the barriers to lean health care implementation. Public Money \& Managemen, v. 31, n. 1, p. 59-66, 2011. http://dx.doi.org/10.1080/09540962.2011.545548

BURGES, N.; RADNOR, Z. Evaluating lean in healthcare. International Journal of Health Care Quality Assurance, v. 26, n. 3, p. 220-235, 2013.

http://dx.doi.org/10.1108/09526861311311418

BURGESS N, RADNOR Z. Service improvement in the English National health service: complexities and tensions. Journal of Management \& Organization, v. 18, n. 5, p. 594-607, 2012. https://doi.org/10.1017/S1833367200000559

CASTALDI, M.; SUGANO, D.; KREPS, K.; CASSIDY, A.; KABAN, J. Lean philosophy and the public hospital. Perioperative Care and Operating Room Management, v. 3, p. 25-28. http://doi.org/10.1016/i.pcorm.2016.05.006

COPETTI, F.A.; SAURIN, T.A.; SOLIMAN, M. Gestão de barreiras na implantação da produção enxuta: um estudo no setor automobilístico. Revista Produção Online, v. 16, n. 1, p. 313-341, 2016. http://dx.doi.org/10.14488/1676-1901.v16i1.2157

DAVIS, J.; ADAMS, J. The 'Releasing Time to Care - the Productive Ward' programme: participants' perspectives. Journal of Nursing Management, v. 20, n. 3, p. 354-360, 2012. http://doi.org/10.1111/j.1365-2834.2011.01266.x

DE CARVALHO, J.C.; RAMOS, M.; PAIXÃO, C. A lean case study in an oncological hospital: implementation of a telephone triage system in the emergency service. Risk Management and Healthcare Policy, v. 7, p. 1-10, 2014. http://doi.org/10.2147/RMHP.S49535

Doğan, N.Ö.; Unutulmaz, O. Lean production in healthcare: a simulation-based value stream mapping in the physical therapy and rehabilitation department of a public hospital. Total Quality Management \& Business Excellence, p. 1-17, 2014. http://dx.doi.org/10.1080/14783363.2014.945312

DROTZ, E.; POKSINSKA, B. Lean in healthcare from employees' perspectives. Journal of Health Organization and Management, v. 28, n. 2, p.177-195, 2014. http://dx.doi.org/10.1108/JHOM-03-2013-0066

EMILIANI, M.L. Supporting small businesses in their transition to lean production. Supply Chain Management: An International Journal, v. 5, n. 2, p. 66-71, 2000. http://dx.doi.org/10.1108/13598540010319975

GIJO, E.V.; ANTONY, J. Reducing Patient Waiting Time in Outpatient Department Using Lean Six Sigma Methodology. Quality and Reliability Engineering International, v. 30, n. 8, p. 1481-1491, 2014. http://doi.org/10.1002/qre.1552

GROVE, A.L.; MEREDITH, J.O.; MACINTYRE, M.; ANGELIS, J.; NEAILEY, K. UK health visiting: challenges faced during lean implementation. Leadership in Health Services, v. 23, n. 3, p. 204-218, 2010. http://dx.doi.org/10.1108/17511871011061037

HAENKE, R.; STICHLER, J.F.; Applying lean six sigma for innovative change to the postanesthesia care unit. Journal of Nursing Administration, v. 45, n. 4, p. 185-187. 2015. http://10.1097/NNA.0000000000000181 
HOLDEN, R.J. Lean thinking in emergency departments: a critical review. Annals of

Emergency Medicine, v. 57, p. 265-278, 2011.

http://dx.doi.org/10.1016/j.annemergmed.2010.08.001

HOLDEN, R.J.; ERIKSSON, A.; ANDREASSON, J.; WILLIAMSSON, A.; DELLVE, L.

Healthcare workers' perceptions of lean: A context-sensitive, mixed methods study in three Swedish hospitals. Applied Ergonomics, v. 47, p. 181-192, 2015.

http://doi.org/10.1016/j.apergo.2014.09.008

KAPLAN, G.S.; PATTERSON, S.H.; CHING, J.M.; BLACKMORE, C.C. Why lean doesn't work for everyone? BMJ Quality \& Safety, July, p. 1-4, 2014.

http://dx.doi.org/10.1136/bmjas-2014-003248

MORODIN, G.A.; FRANK, A.G.; TORTORELLA, G.L.; SAURIN, T.A. Contextual factors and Lean Production implementation in the Brazilian automotive supply chain. Supply Chain

Management: An International Journal, v. 21, n. 4, p. 417-432.

http://dx.doi.org/10.1108/SCM-05-2015-0170

MORROW, E.; ROBERT, G.; MABEN, J.; GRIFFITHS, P. Implementing large scale quality improvement: lessons from The Productive Ward: Releasing Time to Care ${ }^{\mathrm{TM}}$. International Journal of Health Care Quality Assurance, v. 25, n. 4, p. 237-253, 2012.

http://dx.doi.org/10.1108/09526861211221464

POKSINSKA B. The current state of Lean implementation in healthcare: literature review.

Quality Management in Health Care, v. 19, n. 4, p. 319-329, 2010.

http;//doi.org/10.1097/QMH.0b013e3181fa07bb

RADNOR. Z. Implementing lean in health care: making the link between the approach, readiness, and sustainability. International Journal of Industrial Engineering and Management, v. 2, n. 1, p. 1-12, 2011.

RADNOR, Z.J.; HOLWEG, M.; WARING, J. Lean in Healthcare: The unfilled promise?

Social Science \& Medicine, v. 74, p. 364-371, 2012.

http://doi.org/10.1016/j.socscimed.2011.02.011

RADNOR; Z.; WALLEY, P. Learning to walk before we try to run: Adapting lean for the public sector. Public Money \& Management, February, p. 13-20, 2008.

http://dx.doi.org/10.1111/j.1467-9302.2008.00613.x

RINEHART, B. Applying lean principles in healthcare. Radiology Management, v. Suppl, $p$ 19-29, 2013.

RODRIGUES, M.C.N.; PROVIDELLO, M.N.L.; BAGNO, R.B. A influência da engenharia de produção nos serviços de atendimento à saúde: estudo bibliométrico focado em técnicas operacionais. Revista Produção Online, v. 16, n. 1, p. 242-262, 2016.

http://dx.doi.org/10.14488/1676-1901.v16i1.2088

SIM, K.L.; ROGERS, J.W. Implementing lean production systems: barriers to change.

Management Research News, v. 32, n. 1, p. 37-49, 2009.

http://dx.doi.org/10.1108/01409170910922014

TEICH, S.T.; FADDOUL, F.F. Lean management - The journey from Toyota to Healthcare.

Rambam Maimonides Medical Journal, v. 4, n. 2, p. 1-9, 2013.

https://dx.doi.org/10.5041\%2FRMMJ.10107

TEGNER, M.G.; LIMA, P.N.; VEIT, D.R.; NETO, S.L.H.C. Lean office e BPM: proposição e aplicação de método para a redução de desperdícios em áreas administrativas. Revista 
Produção Online, v. 16, n. 3, p. 1007-1032. http://dx.doi.org/10.14488/16761901.v16i3.2308

WOMACK, J.P.; JONES, D.T.; ROOS, D. A máquina que mudou o mundo. Rio de Janeiro. Editora Campus. 2004.

YOUNG, T.P.; MCCLEAN, S.I. A critical look at lean thinking in healthcare. Quality \& Safety in Health Care, v. 17, p. 382-386, 2008. https://doi.org/10.1136/qshc.2006.020131

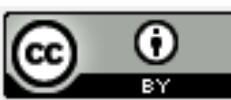

Artigo recebido em 24/10/2016 e aceito para publicação em 20/04/2017

DOI: http://dx.doi.org/10.14488/1676-1901.v17i2.2605 